\title{
Design, Synthesis and Characterization of Benzothiazole Analogues as Promising Pharmacological Agents
}

\author{
Katharigatta Narayanaswamy Venugopala* \\ Department of Biotechnology and Food Technology, Durban University of Technology, Steve Biko Campus, Durban 4001, SOUTH AFRICA.
}

\begin{abstract}
Objective: Benzothiazole moiety containing various functional groups are found to have broad spectrum of biological activity and diverse chemical reactivity. The various pharmacological properties shown by the benzothiazole scaffolds were antitumor, anti-inflammatory, analgesic, antimicrobial, antileishmanial, anticonvulsant, and anti HIV properties. Keeping this in mind a series of (2-(benzo[d]thiazol-2-ylmethoxy)-5-substitutedphenyl) (substitutedphenyl) methanone scaffolds $\mathbf{4 a - f}$ has been designed, synthesized and characterized for suitable pharmacological properties. Methods: A series of (2-(benzo[d]thiazol-2-ylmethoxy)-5-substitutedphenyl) (substitutedphenyl) methanone scaffolds $\mathbf{4 a - f}$ has been synthesized by two steps chemical reactions by conventional stirring method at $40^{\circ} \mathrm{C}$. Purification of the title compounds was achieved by silica gel flash column chromatography method. The characterization of the newly synthesized compounds was achieved by means of $\mathrm{IR}$, NMR $\left({ }^{1} \mathrm{H}\right.$ and $\left.{ }^{13} \mathrm{C}\right)$ and HRMS methods. Results: The yield of the title compounds were found to be satisfactory in the range of $66-79 \%$. Purity of the compounds were found up to $99.36 \%$ by HPLC method. Compounds $\mathbf{4 a}, \mathbf{4 b}, \mathbf{4 c}, \mathbf{4 d}$, and $\mathbf{4 e}$ were studied for single crystal $\mathrm{X}$-ray studies and detailed interactions are reported. Conclusion: Reactions performed to achieve benzothiazole scaffolds $4 \mathbf{4 a - f}$ were environmentally friendly and yielded satisfactory purity and yield. The purified and
\end{abstract}

characterized title compounds are proposed for suitable pharmacological activities in the following communication.

Key words: Benzothiazole analogues, Synthesis, Pharmacological properties, Characterization, Antimosquito, Anti-HIV.

\section{KEY MESSAGE}

The title compounds $\mathbf{4 a - f}$ synthesized by chemical reaction at room temperature was environmentally friendly and yielded satisfactory yield. From the point of pharmacological significance they have been aimed to screen for anti-TB and anti-HIV properties.

Correspondence :

Dr Katharigatta N. Venugopala, Department of Biotechnology and Food Technology, Durban University of Technology, Steve Biko Campus, Durban 4001, SOUTH AFRICA.

Phone no: +27 31373 4887; Fax: +27 862423534

Email: katharigattav@dut.ac.za

DOI: 10.5530/jyp.2017.9.31

\section{INTRODUCTION}

Benzothiazole analogues have been reported for various pharmacological activities, such as those associated with antitumor, ${ }^{1}$ anti-inflammatory, ${ }^{2}$ analgesic, ${ }^{3,4}$ antimicrobial, ${ }^{5,6}$ antileishmanial, ${ }^{7,8}$ antimosquito, ${ }^{9}$ anticonvulsant, ${ }^{10,11}$ and anti-HIV agents. ${ }^{12}$ Keeping all these observations in mind, and in continuation of research on search for cost effective catalysts ${ }^{13,14}$ for the construction of heterocyclic compounds for promising pharmacological properties ${ }^{15-18}$ and method developments, ${ }^{19}$ in the present investigation, it was envisaged for the design and synthesis of a series of (2-(benzo[d]thiazol-2-ylmethoxy)-substitutedphenyl)(4-substitutedphenyl)methanones $4 \mathrm{a}-\mathrm{f}$. The synthesis of the title compounds $4 \mathrm{a}-\mathrm{f}$ was carried out via reaction between 2-(chloromethyl)-benzo[d]-thiazole 2 and 2-hydroxysubstitutedaryl-(substitutedaryl)-methanones 3a-f in dry tetrahydrofuran medium in the presence of potassium carbonate, as depicted in Scheme 1.

\section{METHODS AND MATERIALS}

\section{General chemistry}

The chemicals were procured from Sigma-Aldrich Co. Reactions were monitored using thin-layer chromatography (TLC) and LC-MS. TLC was performed on Merck 60 F-254 silica gel plates with visualization by ultraviolet (UV) light using ethyl acetate: $n$-hexane as a solvent system. The melting points were determined on a Büchi Melting Point B-545 apparatus (BÜCHI Labortechnik AG, Flawil, Switzerland). The IR spectra were recorded on a Nicolet 6700 Fourier transform infrared (FT-IR)

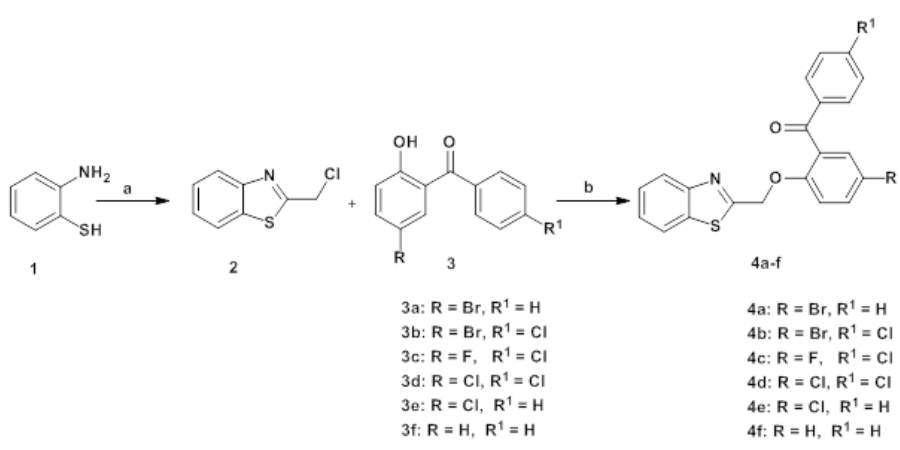

Scheme 1: Synthetic scheme for the construction of 4a-f. Reagents and conditions: a) chloroacetic acid, polyphosphoric acid, $8 \mathrm{~h}$ reflux; b) $\mathrm{K}_{2} \mathrm{CO}_{3^{\prime}}$ dry $\mathrm{THF}, 40^{\circ} \mathrm{C}$, overnight.

spectrometer (Thermo Fisher Scientific, Waltham, MA, USA). ${ }^{1} \mathrm{H}$ and ${ }^{13} \mathrm{C}$ NMR spectra were recorded on Bruker AVANCE III $400 \mathrm{MHz}$ instruments (Bruker Corporation, Billerica, MA, USA) with $\mathrm{CDCl}_{3}$ as a solvent. Chemical shifts $(\delta)$ were indicated in parts per million downfield from tetramethylsilane, and the coupling constants $(J)$ were recorded in Hertz. The splitting pattern is abbreviated as follows: s, singlet; $\mathrm{d}$, doublet; m, multiplet. Mass spectra were recorded using the LC-MSAgilent 1100 series (Agilent Technologies, Santa Clara, CA, USA) with a mass selective detector (MSD) (ion trap) using 0.1\% aqueous trifluoroacetic acid (TFA) in an acetonitrile system on a C18-BDS column for a

This is an open access article distributed under the terms of the Creative Commons Attribution-NonCommercial-ShareAlike 4.0 License, which allows others to remix, tweak, and build upon the work non-commercially, as long as the author is credited and the new creations are licensed under the identical terms. 
Venugopala: Benzothiazole Analogues as Promising Pharmacological Agents

duration of 10 minutes. HRMS were acquired using a Bruker MicroToF Q11 time-of-flight mass spectrometer (Bruker Corporation). ClogP of the title compounds was determined using ChemBioDraw Ultra v. 13.0 (PerkinElmer, Inc.). The purity of the compounds was assessed using the high-performance liquid chromatography (HPLC) method.

\section{Procedure involved in the synthesis of 2-(chloromethyl)-benzo[d]-} thiazole (2)

A mixture of chloroaceticacid $(3.72 \mathrm{~g}, 0.040 \mathrm{~mol})$ and polyphosphoric acid $(8 \mathrm{~g})$ was heated to $180^{\circ} \mathrm{C}$ and 2 -aminobenzenethiol $(4 \mathrm{~g}, 0.032 \mathrm{~mol})$ was added and stirred at reflux for $8 \mathrm{~h}$. After cooling, the reaction mixture was basified with $5 \mathrm{~N}$ sodium hydroxide and the solution was extracted with chloroform ( 4 x $20 \mathrm{~mL})$. The organic layer was dried over sodium sulfate and removed under vacuum. The residue obtained was purified by column chromatography on silica gel using ethyl acetate and $n$-hexane as an eluent to yield yellow oil in $61.27 \%$ yield. ${ }^{1} \mathrm{H}$ NMR (400 $\left.\mathrm{MHz} \mathrm{CDCl}_{3}\right)$ ): $\delta=4.96\left(\mathrm{~s}, 2 \mathrm{H}, \mathrm{OCH}_{2}\right), 7.41-7.54(\mathrm{~m}, 2 \mathrm{H}, \mathrm{Ar}-\mathrm{H})$, 7.90-7.92 (m, 1H, Ar-H), 8.02-8.05 (m, 1H, Ar-H). LC-MS: m/z 183.6 $\left(\mathrm{M}^{+}\right)$. Anal. calcd. for $\mathrm{C}_{8} \mathrm{H}_{6} \mathrm{ClNS}$ : C, 52.32; H, 3.29; N, 7.63 Found C, 52.22; $\mathrm{H}, 3.31 ; \mathrm{N}, 7.69$.

General procedure for the synthesis of (2-(benzo[d] thiazol-2-ylmethoxy)substitutedphenyl)(4-substitutedphenyl)methanone (4a-f)

To a solution of 2-(chloromethyl)-benzo[d]-thiazole $(0.5 \mathrm{~g}, 0.0027 \mathrm{~mol})$ and (2-hydroxysubstitutedphenyl) (substitutedphenyl) methanone $(0.0027 \mathrm{~mol})$ in dry THF, dry potassium carbonate $(0.380 \mathrm{~g}, 0.0027 \mathrm{~mol})$ was added and stirred at $40^{\circ} \mathrm{C}$ overnight. The reaction mixture was concentrated to remove solvent and diluted with ethyl acetate, washed with water, brine solution and dried over anhydrous sodium sulfate. The organic layer was concentrated to yield residue and purified by silica gel flash column chromatography using ethyl acetate and $n$-hexane as eluent.

(2-(benzo[d]thiazol-2-ylmethoxy)-5-bromophenyl)(phenyl)methanone (4a) A white solid $\left(R_{\mathrm{f}}=0.71\right)$. IR $\left(\mathrm{cm}^{-1}\right): 3061(\mathrm{ArC}-\mathrm{H}), 1654(\mathrm{C}=\mathrm{O}), 1584$ $(\mathrm{C}=\mathrm{N}), 1523,1475(\mathrm{C}=\mathrm{C}), 662(\mathrm{C}-\mathrm{Br}) .{ }^{1} \mathrm{H}$ NMR $\left(400 \mathrm{MHz}, \mathrm{CDCl}_{3}\right)$ : $\delta=5.36\left(\mathrm{~s}, 2 \mathrm{H}, \mathrm{OCH}_{2}\right), 6.95-6.98(\mathrm{~d}, J=8.00 \mathrm{~Hz}, 1 \mathrm{H}), 7.32-7.36(\mathrm{~m}, 1 \mathrm{H})$, 7.42-7.46 (m, 3H), 7.52-7.56 (m, 3H), 7.76-7.84 (m, 3H), 7.93-7.95 $(\mathrm{d}, J=8.12 \mathrm{~Hz}, 1 \mathrm{H}) .{ }^{13} \mathrm{C} \mathrm{NMR}\left(400 \mathrm{MHz}, \mathrm{CDCl}_{3}\right): \delta=68.72,114.61$, $114.84,121.96,123.24,125.51,126.39,128.74,130.10,131.46,132.66$, $133.70,134.77,135.26,137.40,152.87,154.55,167.34,194.49$. HRMS calculated for $\mathrm{C}_{21} \mathrm{H}_{15} \mathrm{BrNO}_{2} \mathrm{~S}\left(\mathrm{M}+\mathrm{H}^{+}\right) 424.0001$ found 424.0014 .

(2-(benzo[d]thiazol-2-ylmethoxy)-5-bromophenyl)(4-chlorophenyl) methanone (4b)

A brown solid $\left(R_{\mathrm{f}}=0.75\right)$. IR $\left(\mathrm{cm}^{-1}\right): 3068(\mathrm{ArC}-\mathrm{H}), 1653(\mathrm{C}=\mathrm{O}), 1584$ $(\mathrm{C}=\mathrm{N}), 1520,1488(\mathrm{C}=\mathrm{C}), 756(\mathrm{C}-\mathrm{Cl}), 528(\mathrm{C}-\mathrm{Br}) .{ }^{1} \mathrm{H}$ NMR $(400 \mathrm{MHz}$, $\left.\mathrm{CDCl}_{3}\right): \delta=5.37\left(\mathrm{~s}, 2 \mathrm{H}, \mathrm{OCH}_{2}\right), 6.96-6.99(\mathrm{~m}, 1 \mathrm{H}), 7.35-7.56(\mathrm{~m}, 6 \mathrm{H})$, 7.75-7.81 (m, 3H), 7.94-7.96 (d, $J=8.08 \mathrm{~Hz}, 1 \mathrm{H}) .{ }^{13} \mathrm{C} \mathrm{NMR}(400 \mathrm{MHz}$, $\left.\mathrm{CDCl}_{3}\right): \delta=68.70,114.76,114.93,122.06,123.40,125.70,126.55,129.18$, $130.98,131.48,132.77,135.22,135.91,140.33,152.96,154.57,166.97$, 193.39. HRMS calculated for $\mathrm{C}_{21} \mathrm{H}_{14} \mathrm{BrClNO}_{2} \mathrm{~S}\left(\mathrm{M}+\mathrm{H}^{+}\right) 457.9612$ found 457.9598 .

\section{(2-(benzo[d]thiazol-2-ylmethoxy)-5-fluorophenyl)(4-chlorophenyl) methanone (4c)}

A yellow solid $\left(R_{\mathrm{f}}=0.70\right)$. IR $\left(\mathrm{cm}^{-1}\right): 3068(\mathrm{ArC}-\mathrm{H}), 1653(\mathrm{C}=\mathrm{O})$, $1584(\mathrm{C}=\mathrm{N}), 1520,1488(\mathrm{C}=\mathrm{C}), 1087(\mathrm{C}-\mathrm{F}), 756(\mathrm{C}-\mathrm{Cl}) .{ }^{1} \mathrm{H}$ NMR $\left(400 \mathrm{MHz}, \mathrm{CDCl}_{3}\right): \delta=5.35\left(\mathrm{~s}, 2 \mathrm{H}, \mathrm{OCH}_{2}\right), 7.02-7.06(\mathrm{~m}, 1 \mathrm{H}), 7.13-$ $7.15(\mathrm{~m}, 2 \mathrm{H}), 7.34-7.47(\mathrm{~m}, 4 \mathrm{H}), 7.64-7.81(\mathrm{~m}, 3 \mathrm{H})$, 7.94-7.96 (m. 1H). ${ }^{13} \mathrm{C} \mathrm{NMR}\left(400 \mathrm{MHz}, \mathrm{CDCl}_{3}\right): \delta=69.18,114.68,116.85,118.71,122.02$, $123.30,125.62,126.47,129.09,131.44,135.22,135.85,140.17,151.58$,
$152.88,156.46,158.88,167.28,193.51$. HRMS calculated for $\mathrm{C}_{21} \mathrm{H}_{14} \mathrm{ClF}-$ $\mathrm{NO}_{2} \mathrm{~S}\left(\mathrm{M}+\mathrm{H}^{+}\right) 398.0412$ found 398.0413 .

(2-(benzo[d]thiazol-2-ylmethoxy)-5-chlorophenyl)(4-chlorophenyl) methanone (4d)

A brown solid $\left(R_{\mathrm{f}}=0.73\right)$. IR $\left(\mathrm{cm}^{-1}\right): 3062($ ArC-H $), 1652(\mathrm{C}=\mathrm{O}), 1592$ $(\mathrm{C}=\mathrm{N}), 1519,1478(\mathrm{C}=\mathrm{C}), 755(\mathrm{C}-\mathrm{Cl}) .{ }^{1} \mathrm{H} \mathrm{NMR}\left(400 \mathrm{MHz}, \mathrm{CDCl}_{3}\right)$ : $\delta=5.37\left(\mathrm{~s}, 2 \mathrm{H}, \mathrm{OCH}_{2}\right), 7.01-7.04(\mathrm{~d}, J=8.64 \mathrm{~Hz}, 1 \mathrm{H}), 7.37-7.48(\mathrm{~m}, 6 \mathrm{H})$, 7.76-7.81 (m, 3H), 7.94-7.96 (d, $J=8.12 \mathrm{~Hz}, 1 \mathrm{H}) .{ }^{13} \mathrm{C}$ NMR $(400 \mathrm{MHz}$, $\left.\mathrm{CDCl}_{3}\right): \delta=68.79,114.50,122.03,123.33,125.68,126.51,127.63,129.11$, $129.93,130.54,131.43,132.17,135.22,135.87,140.21,152.87,154.01$, 166.98, 193.44. HRMS calculated for $\mathrm{C}_{21} \mathrm{H}_{14} \mathrm{Cl}_{2} \mathrm{NO}_{2} \mathrm{~S}\left(\mathrm{M}+\mathrm{H}^{+}\right) 414.0117$ found 414.0118 .

(2-(benzo[d]thiazol-2-ylmethoxy)-5-chlorophenyl)(phenyl)methanone (4e) A white solid $\left(R_{\mathrm{f}}=0.68\right)$. IR $\left(\mathrm{cm}^{-1}\right): 3062(\mathrm{ArC}-\mathrm{H}), 1653(\mathrm{C}=\mathrm{O}), 1593$ $(\mathrm{C}=\mathrm{N}), 1523,1477(\mathrm{C}=\mathrm{C}), 758(\mathrm{C}-\mathrm{Cl}) .{ }^{1} \mathrm{H}$ NMR $\left(400 \mathrm{MHz}, \mathrm{CDCl}_{3}\right)$ : $\delta=5.37\left(\mathrm{~s}, 2 \mathrm{H}, \mathrm{OCH}_{2}\right), 7.01-7.03(\mathrm{~m}, 1 \mathrm{H}), 7.32-7.46(\mathrm{~m}, 6 \mathrm{H}), 7.53-$ $7.56(\mathrm{~m}, 1 \mathrm{H}), 7.77-7.85(\mathrm{~m}, 3 \mathrm{H}), 7.93-7.95(\mathrm{~d}, 8.16 \mathrm{~Hz}, 1 \mathrm{H}) .{ }^{13} \mathrm{C} \mathrm{NMR}$ $\left(400 \mathrm{MHz}, \mathrm{CDCl}_{3}\right): \delta=68.82,114.47,121.98,123.25,125.52,126.40$, $127.47,128.76,129.87,130.12,131.08,131.83,133.72,135.27,137.43$, $152.89,154.05,167.43,194.64$. HRMS calculated for $\mathrm{C}_{21} \mathrm{H}_{15} \mathrm{ClNO}_{2} \mathrm{~S}$ $\left(\mathrm{M}+\mathrm{H}^{+}\right) 380.0507$ found 380.0502 .

\section{(2-(benzo[d]thiazol-2-ylmethoxy)phenyl)(phenyl)methanone (4f)}

A brown solid $\left(R_{\mathrm{f}}=0.61\right)$. IR $\left(\mathrm{cm}^{-1}\right): 3062($ ArC-H $), 1653(\mathrm{C}=\mathrm{O}), 1593$ $(\mathrm{C}=\mathrm{N}), 1477(\mathrm{C}=\mathrm{C}), 758(\mathrm{C}-\mathrm{Cl}) .{ }^{1} \mathrm{H} \mathrm{NMR}\left(400 \mathrm{MHz}, \mathrm{CDCl}_{3}\right): \delta=5.41$ $\left(\mathrm{s}, 2 \mathrm{H}, \mathrm{OCH}_{2}\right), 7.03-7.06(\mathrm{~m}, J=9.51 \mathrm{~Hz}, 1 \mathrm{H}), 7.26-7.50(\mathrm{~m}, 6 \mathrm{H}), 7.56-$ $7.60(\mathrm{~m}, 1 \mathrm{H}), 7.80-7.88(\mathrm{~m}, 3 \mathrm{H}), 7.97-7.99(\mathrm{~d}, 8.07 \mathrm{~Hz}, 1 \mathrm{H}) .{ }^{13} \mathrm{C} \mathrm{NMR}$ $\left(400 \mathrm{MHz}, \mathrm{CDCl}_{3}\right): \delta=68.59,114.31,121.81,123.16,125.43,126.29$, $127.31,128.67,129.78,130.08,131.01,131.66,133.64,135.18,137.37$, $152.75,154.00,167.42,194.57$. HRMS calculated for $\mathrm{C}_{21} \mathrm{H}_{15} \mathrm{NO}_{2} \mathrm{~S}$ $\left(\mathrm{M}+\mathrm{H}^{+}\right) 346.0721$ found 346.0722 .

\section{RESULTS AND DISCUSSION}

\section{Chemistry}

The 2-hydroxysubstituted aryl-(substitutedaryl)-methanones 3a-f were prepared according to a procedure described in the literature. ${ }^{20}$ Conversely, 2-(chloromethyl)-benzo[d]-thiazole 2 was synthesized from the equimolar reactions of 2-aminobenzenethiol 1 and 2-chloroacetic acid in the presence of polyphosphoric acid according to the described procedure. ${ }^{21}$ The title compounds $4 \mathrm{a}-\mathrm{f}$ were characterized by infrared (IR) spectroscopy, nuclear magnetic resonance (NMR) $\left({ }^{1} \mathrm{H}\right.$ and $\left.{ }^{13} \mathrm{C}\right)$, and high-resolution mass spectrometry (HRMS). The yield and purity of compounds $4 \mathrm{a}-\mathrm{f}$ were found to be in the range of $66-79$ and $>99.36 \%$, respectively. The physicochemical characteristics of the title compounds are reported in Table 1 . In the proton NMR spectrum of an intermediate 2-(chloromethyl)-benzo[d]-thiazole (2), the methylene protons are observed at $\delta 4.96$ and the molecular mass of the compound is in agreement with the molecular ion peak of $183.6\left(\mathrm{M}^{+}\right)$on liquid chromatographymass spectrometry (LC-MS). The IR spectra of the title compounds $\{2$-(benzo[d]-thiazol-2-yl-methoxy)-substitutedaryl\}-(substitutedaryl)methanones $4 \mathrm{a}-\mathrm{f}$ exhibited characteristic carbonyl stretching in the range of $1651-1654 \mathrm{~cm}^{-1}$. The proton NMR spectra of compounds $4 \mathrm{a}-\mathrm{f}$ exhibited singlet methylene protons in the range of $\delta$ 5.39-5.41 and carbonyl carbon at $\delta 193.01-194.33$ in the ${ }^{13} \mathrm{C}$ NMR spectra as well. With respect to HRMS, the molecular ion peaks of title compounds $4 \mathrm{a}-\mathrm{f}$ were in compliance with the proposed molecular weight. The $\operatorname{Clog} P$ value of the title compounds $4 \mathrm{a}-\mathrm{f}$ was calculated using the ChemBioDraw Ultra software (v. 13.0; PerkinElmer, Inc., Waltham, MA, USA) and the values were in the range of 3.9589-6.7397. It was shown that heterocyclic compounds tend to exhibit polymorphic, ${ }^{22}$ pseudopolymorphic, ${ }^{23}$ and 
Table 1: Physicochemical characteristics of 2-substituted benzothiazole analogues $4 a-f$

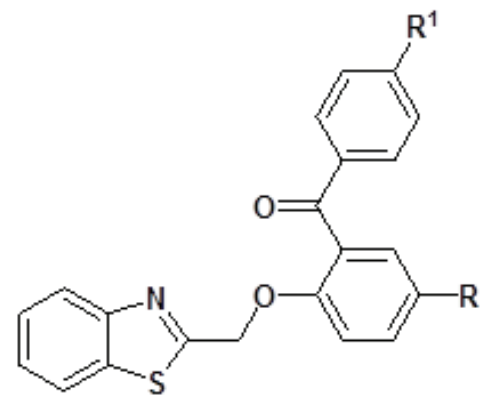

\begin{tabular}{ccccccc}
\hline Compound & $\mathbf{R}$ & $\mathbf{R}^{1}$ & $\mathbf{M} . \mathbf{F}$ (M. Wt.) & $\begin{array}{c}\text { Yield } \\
(\%)^{\mathbf{a}}\end{array}$ & m.p.( $\left.{ }^{\circ} \mathrm{C}\right)$ & $\mathbf{C l o g}^{\mathrm{b}}$ \\
\hline $4 \mathrm{a}$ & $\mathrm{Br}$ & $\mathrm{H}$ & $\begin{array}{c}\mathrm{C}_{21} \mathrm{H}_{14} \mathrm{BrNO}_{2} \mathrm{~S} \\
(422.9929)\end{array}$ & 77.62 & $133-134$ & 6.0107 \\
$4 \mathrm{~b}$ & $\mathrm{Br}$ & $\mathrm{Cl}$ & $\begin{array}{c}\mathrm{C}_{21} \mathrm{H}_{13} \mathrm{BrClNO}_{2} \mathrm{~S} \\
(456.9539)\end{array}$ & 64.00 & $177-178$ & 6.7397 \\
$4 \mathrm{c}$ & $\mathrm{F}$ & $\mathrm{Cl}$ & $\begin{array}{c}\mathrm{C}_{21} \mathrm{H}_{13} \mathrm{ClFNO}_{2} \mathrm{~S} \\
(397.0340)\end{array}$ & 78.05 & $146-147$ & 6.0541 \\
$4 \mathrm{~d}$ & $\mathrm{Cl}$ & $\mathrm{Cl}$ & $\begin{array}{c}\mathrm{C}_{21} \mathrm{H}_{13} \mathrm{Cl}_{2} \mathrm{NO}_{2} \mathrm{~S} \\
(413.0044)\end{array}$ & 83.21 & $175-176$ & 6.5897 \\
$4 \mathrm{e}$ & $\mathrm{Cl}$ & $\mathrm{H}$ & $\begin{array}{c}\mathrm{C}_{21} \mathrm{H}_{14} \mathrm{ClNO}_{2} \mathrm{~S} \\
(379.0434)\end{array}$ & 70.80 & $130-131$ & 4.7137 \\
$4 \mathrm{f}$ & $\mathrm{H}$ & $\mathrm{H}$ & $\begin{array}{c}\mathrm{C}_{21} \mathrm{H}_{15} \mathrm{NO}_{2} \mathrm{~S} \\
(345.0823)\end{array}$ & 68.21 & $112-113$ & 3.9589 \\
\hline
\end{tabular}

${ }^{a}$ All the yields are on isolated basis. Purified by silica gel flash column chromatography employing ethyl acetate: $\mathrm{n}$-hexane (7:3) as solvent system.

${ }^{\mathrm{b}} \mathrm{C} \log P$ was calculated using ChemBioDraw Ultra software v13.0.

concomitant polymorphic ${ }^{24}$ behavior. Keeping this in mind, an attempt was made to screen the title compounds $4 \mathrm{a},{ }^{25} 4 \mathrm{~b},{ }^{26} 4 \mathrm{c},{ }^{27} 4 \mathrm{~d},{ }^{28}$ and $4 \mathrm{e}^{29}$ for polymorphic behavior using single crystal X-ray studies; none of the compounds exhibited polymorphic behavior.

\section{CONCLUSION}

Title compounds $\{2$-(benzo[d]-thiazol-2-yl-methoxy)-substitutedaryl $\}$ (substitutedaryl)-methanones $\mathbf{4 a - f}$ have been synthesized by two steps chemical reaction. The yield and purity of the compounds were found to be satisfactory. The title compounds are further aimed to screen for suitable pharmacological properties.

\section{ACKNOWLEDGEMENT}

The author is grateful to Deanship of Scientific Research, King Faisal University, Kingdom of Saudi Arabia for the support (grant number 160008) and encouragement.

\section{CONFLICT OF INTEREST}

No conflict of interest are declared.

\section{ABBREVIATION USED}

FT-IR: Fourier transform infrared; NMR: Nuclear magnetic resonance; HRMS: High resolution mass spectrometry; TLC: Thin layer chroma- tography; LC-MS: Liquid chromatography-mass spectrometry; HPLC: High-performance liquid chromatography.

\section{REFERENCES}

1. Yoshida M, Hayakawa C, Hayashi N, Agatsuma T, Oda Y, Tanzawa F, et al. Synthesis and biological evaluation of benzothiazole derivatives as potent antitumor agents. Bioorg Med Chem Lett. 2005;15(14):3328-32.

2. Paramashivappa R, Kumar PP, Rao PVS, Rao AS. Design, synthesis and biological evaluation of benzimidazole/benzothiazole and benzoxazole derivatives as cyclooxygenase inhibitors. Bioorg Med Chem Lett. 2003;13(4):657-60.

3. Baell JB, Forsyth SA, Gable RW, Norton RS, Mulder RJ. Design and synthesis of type-III mimetics of omega-conotoxin GVIA. J Comput Aided Mol Des. 2001;15(12):1119-36

4. Westaway SM, Thompson M, Rami HK, Stemp G, Trouw LS, Mitchell DJ, et al. Design and synthesis of 6-phenylnicotinamide derivatives as antagonists of TRPV1. Bioorg Med Chem Lett. 2008;18(20):5609-13.

5. Koci J, Klimesova V, Waisser K, Kaustova J, Dahse HM, Mollmann U. Heterocyclic benzazole derivatives with antimycobacterial in vitro activity. Bioorg Med Chem Lett. 2002;12(22):3275-8.

6. Alang G, Kaur G, Kaur R, Singh A, Tiwari R. Synthesis, Characterization, and Biological Evaluation of certain 6-methyl-2(3H)-benzo-1, 3-thiazolyl-1'-ethylidene-2-(o, p-Substituted Acetophenones) Hydrazine Analogs. J Young Pharm. 2010;2(4):394-8

7. Delmas F, Avellaneda A, Giorgio CD, Robin M, Clercq ED, Timon-David $P$, et al Synthesis and antileishmanial activity of (1,3-benzothiazol-2-yl) amino-9-(10h)acridinone derivatives. Eur J Med Chem. 2004;39(8):685-90.

8. Delmas F, Giorgio CD, Robin M, Azas N, Gasquet M, Detang C, et al. In vitro activities of position 2 substitution-bearing 6-nitro- and 6-amino-benzothiazoles and their corresponding anthranilic acid derivatives against Leishmania infantum and Trichomonas vaginalis. Antimicrob Agents Chemother. 2002;46(8):2588-94.

9. Venugopala KN, Krishnappa M, Nayak SK, Subrahmanya BK, Vaderapura JP, Chalannavar RK, et al. Synthesis and antimosquito properties of 2,6-substituted benzo[ $d]$ thiazole and 2,4-substituted benzo[d]thiazole analogues against Anopheles arabiensis. Eur J Med Chem. 2013;65:295-303.

10. Priyanka C, Pramod KS, Anjana S, Jonish V. Recent advances in pharmacological activity of benzothiazole derivatives. Int J Current Pharm Res. 2010;2(4):5-11.

11. Ucar $H$, Van derpoorten $K$, Cacciaguerra $S$, Spampinato $S$, Stables JP, Depovere $P$, et al. Synthesis and anticonvulsant activity of 2(3H)-benzoxazolone and 2(3H)benzothiazolone derivatives. J Med Chem. 1998;41(7):1138-45.

12. Racane L, Tralic-Kulenovic V, Fiser-Jakic L, Boykin DW, Karminski-Zamola G. Synthesis of bis-substituted amidinobenzothiazoles as potential anti-HIV agents. Heterocycles. 2001;55(11):2085-98.

13. Venugopala KN, Prasanna RT, Odhav B. Trifluoroacetic acid: An efficient catalyst for paal-knorr pyrrole synthesis and its deprotection. Asian J Chem. 2013:25(15):8685

14. Chandrashekharappa S, Venugopala KN, Venugopala R, Odhav B. Silica-Sulfuric Acid: Novel, Simple, Efficient and Reusable Catalyst for Hydration of Nitrile to Amide. Asian J Chem. 2016;28(10):2177.

15. Kasumbwe K, Venugopala K, Mohanlall V, Odhav B. Synthetic mono/di-halogenated coumarin derivatives and their anticancer properties. Anticancer Agents Med Chem. 2016.

16. Venugopala KN, Rao GD, Bhandary S, Pillay M, Chopra D, Aldhubiab BE, et al. Design, synthesis, and characterization of (1-(4-aryl)-1 H-1, 2, 3-triazol-4-yl) methyl, substituted phenyl-6-methyl-2-oxo-1，2，3，4-tetrahydropyrimidine-5-carboxylates against Mycobacterium tuberculosis. Drug Des Devel Ther. 2016;10:2681.

17. Sandeep C, Venugopala KN, Gleiser RM, Chetram A, Padmashali B, Kulkarni RS et al. Greener synthesis of indolizine analogues using water as a base and solvent: study for larvicidal activity against Anopheles arabiensis. Chem Biol Drug Des. 2016;88(6):899-904.

18. Venugopala KN, Gleiser M R, Chalannavar KR, Odhav B. Antimosquito properties of 2-substituted phenyl/benzylamino-6-(4-chlorophenyl)-5-methoxycarbonyl4-methyl-3, 6-dihydropyrimidin-1-ium chlorides against Anopheles arabiensis. Med Chem. 2014;10(2):211-9.

19. Venugopala KN, Jayashree BS. Microwave-induced synthesis of Schiff bases of aminothiazolyl bromocoumarins as antibacterials. Indian J Pharm Sci. 2008;70(1):88.

20. Tawada $H$, Natsugari $H$, Ishikawa E, SugiyamaY, Ikeda $H$, Meguro K. Synthesis of 3-ureido derivatives of coumarin and 2-quinolone as potent acyl-CoA:cholesterol acyltransferase inhibitors. Chem Pharm Bull. 1995;43(4):616-25

21. Gellis A, Boufatah N, Vanelle P. Rapid microwave-promoted synthesis of new sulfonylmethylbenzothiazoles in water. Green Chem. 2006;8(5):483-7.

22. Nayak SK, Venugopala KN, Chopra D, Row TNG. Insights into conformational and packing features in a series of aryl substituted ethyl-6-methyl-4-phenyl-2-oxo1,2,3,4-tetrahydropyrimidine-5-carboxylates. CrystEngComm. 2011;13(2):591-605

23. Panini P, Venugopala KN, Odhav B, Chopra D. Polymorphism in two biologically active dihydropyrimidinium hydrochloride derivatives: quantitative inputs towards the energetics associated with crystal packing. Acta Crystallogr Sect B. $2014 ; 70(4): 681-96$. 
24. Munshi P, Venugopala KN, Jayashree BS, Guru Row TN. Concomitant polymorphism in 3-acetylcoumarin: Role of weak $\mathrm{C}-\mathrm{H} \cdots \mathrm{O}$ and $\mathrm{C}-\mathrm{H} \cdots \pi$ interactions. Cryst Growth Des. 2004;4(6):1105-7.

25. Venugopala KN, Nayak SK, Odhav B. \{2-[(1,3-Benzothiazol-2-yl)methoxy]-5-bromophenyl\}(phenyl)methanone. Acta Crystallogr Sect E. 2013;69(Pt 6):0984-05.

26. Nayak SK, Venugopala KN, Govender T, Kruger HG, Maguire GEM. [2-(1,3-Benzothiazol-2-ylmethoxy)-5-bromophenyl](4-chlorophenyl)methanone. Acta Crystallogr Sect E. 2013;69(Pt 1):070-o1.
27. Venugopala KN, Nayak SK, Govender T, Kruger HG, Maguire GEM. \{2-[(1,3-Benzothiazol-2-yl)methoxy]-5-fluorophenyl\}(4-chlorophenyl)methanone. Acta Crystallogr Sect E. 2013;69(Pt 7):01007-08.

28. Venugopala KN, Nayak SK, Odhav B. \{2-[(1,3-Benzothiazol-2-yl)methoxy]5-chlorophenyl\}(4-chlorophenyl)methanone. Acta Crystallogr Sect E. 2013;69 (Pt 7):01124-0.

29. Venugopala KN, Nayak SK, Govender T, Kruger HG, Maguire GEM. (2-(Benzo[d] thiazol-2yl-methoxy)-5-chlorophenyl)(phenyl)methanone. Acta Crystallogr Sect

Article History: Submission Date: 09-02-17; Received Date: 15-03-17; Acceptance Date: 20-03-2017.

Cite this article: Venugopala KN. Design, Synthesis and Characterization of Benzothiazole Analogues as Promising Pharmacological Agents. J Young Pharm. 2017:9(2):158-161. 